\title{
"Capture and Release of the Chthonic Beasts": Archaeological Heritage as Digital Commons in Contemporary Art Practice. Various Thoughts on the Occasion of the Artwork 'Future Bestiary'
}

\author{
Marina Markellou and Petros Moris
}

This chapter is a formal attempt at an unstructured informal exchange of opinions and thoughts between Marina and Petros on the occasion of Petros' art show titled 'Future Bestiary'. Through this specific case study, the aesthetic, artistic and ethical impact of contemporary artistic practices that transgress the traditional notions of originality and authenticity were explored, while questions about how digital creations are controlled through Copyright Law and the Archaeological Law and how Intellectual Property is managed were also raised. The rise of contemporary art practices such as that of Petros', clearly inspired by archaeological heritage, produces unprecedented and unusual digital reconstructions through prompts to reconsider the fundamental structure of traditional legal systems and move towards an alternative legal framework that enables creativity in a collaborative fashion.

How to cite this book chapter:

Markellou, M. and Moris, P. 2020. "Capture and Release of the Chthonic Beasts": Archaeological Heritage as Digital Commons in Contemporary Art Practice. Various Thoughts on the Occasion of the Artwork 'Future Bestiary'. In Lekakis, S. (ed.) Cultural Heritage in the Realm of the Commons: Conversations on the Case of Greece. Pp. 127-140. London: Ubiquity Press. DOI: https://doi.org/10.5334/bcj.h. License: CC-BY 

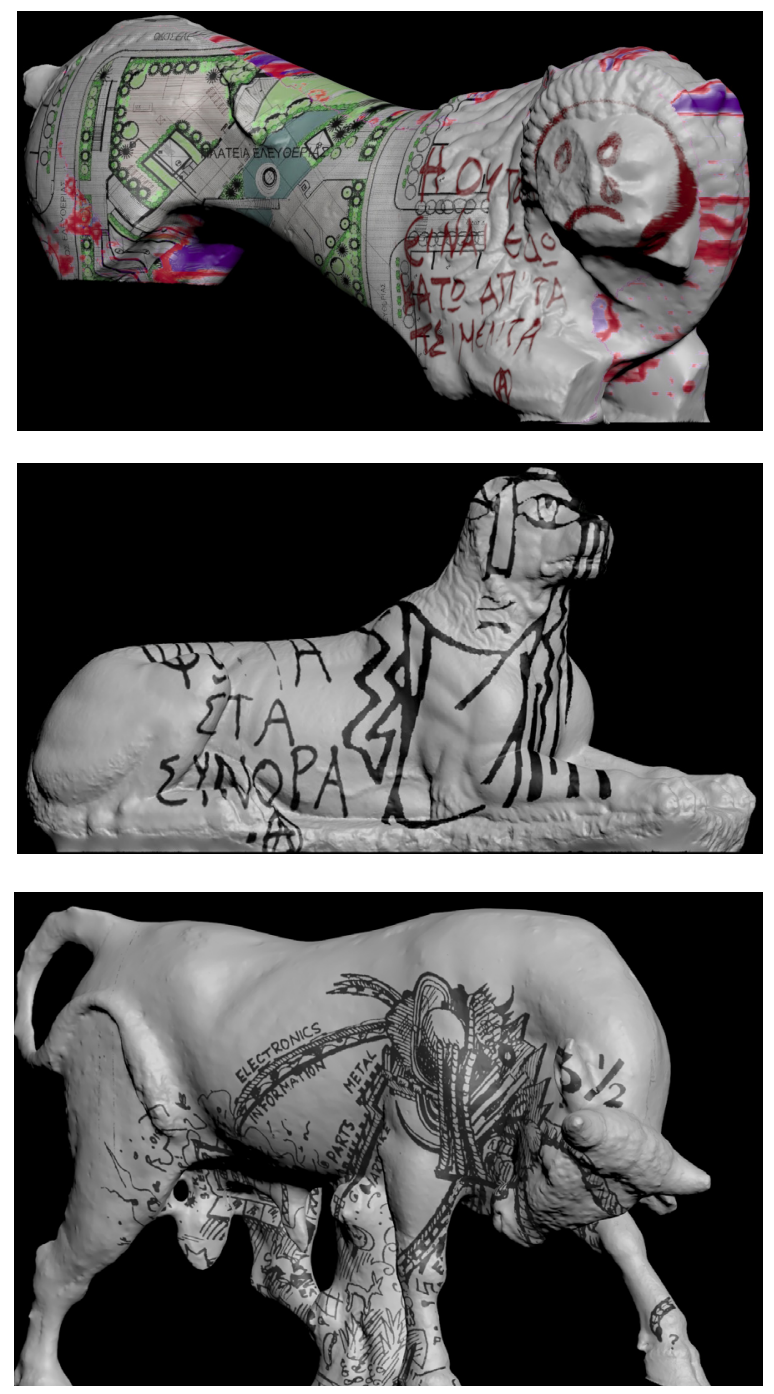

Figures 1, 2, 3: Petros Moris, Future Bestiary, 2019, HD video projection (5:00) (Source: PM).

\section{Future Bestiary}

The work 'Future Bestiary' is a video-projection that could be perceived as a type of fragmented, open-ended visual essay. The primary material that makes up this digitally animated narrative was gathered by Petros during an autumn afternoon around the idyllic archaeological site of Kerameikos ancient cemetery, and its adjacent elegant museum in the centre of Athens. 
The primary material of 'Future Bestiary' is a series of photogrammetric documentations, rendered within the video as three-dimensional digital forms. The original subjects of these forms come from the funerary sculptures of Kerameikos. More specifically, they focus on the mythical and naturalistic animal sculptures that once adorned the ancient cemetery, inducing depictions of a molossian hound, sphinxes, lions and a mighty bull. This system of sculptural forms is understood here as a type of chthonic bestiary, which is lined up sequentially throughout the video in the form of rotating three-dimensional depictions. In the video, these perpetually rotating digital surfaces, which emerged by means of the photogrammetric process, become the 'canvas' for the inclusion of visual elements recovered online. These elements find their way onto the $3 \mathrm{D}$ reconstructions via an 'intrusive' style, simulating immaterial graffiti, tattoos, talismans or other graphic typologies.

In this way, the digital reconstructions of the ancient forms become a system of mnemonic ethereal bodies, onto which the fantasies and mythologies that concern realities of the present and speculations of the future are inscribed. More specifically, these are prospects, fears and desires that concern the future of the city of Athens, a future which is evoked here through references to urban development projects, technological innovations, algorithmic economic systems, social upheavals and cultural metamorphoses. Beyond providing an informational and narrative layer that orchestrates the conceptual premise and aesthetic character of the work, this series of visual projections functions inevitably as an 'iconoclastic' gesture, a simulated 'defacement'. As is the case with the non-destructive technique of documentation and reproduction involved in photogrammetry, this equally immaterial, non-destructive gesture further hybridises the perplexed status of these digital clones of archaeological remains, of this 'captured' and 'released' cultural material. It also triggers a further exploration of the essence and affordances of cultural heritage in the Greek context.

\section{The photogrammetric technique and its affordances}

If we set aside this additional layer of artistic conceptual and formal remix for a moment, we can see that, for a significant part, the complications that can be found in the way 'Future Bestiary' deals with material cultural heritage lie in the nature of the photogrammetric technique itself. Photogrammetry is a non-destructive technology used to derive accurate 3D metric and descriptive object information from photographs. It is a well-established technique for archaeological documentation and cultural heritage conservation, as it provides a precise method of acquiring three-dimensional information relating to sculptures, cultural monuments and historical sites (Al-Ruzouq 2012). In recent years, photogrammetric processing has been used as a basis for further analysis and interpretation of cultural goods from an artistic perspective. 
There is a telling semiology in the alternative terminology for the technique of photogrammetry, known as Structure-from-Motion. As its most common applications would suggest, one can imagine a bodily and active practice, a process of corporeal motion around objects, involving a series of repetitive photographic shots from different angles. This fundamentally 'analogue' element of human motion, bound always to physical and cultural aspects, introduces us to an overall set of particular, non-quantifiable attributes, a series of complexities of the photogrammetric practice.

Indeed, in contrast to other hi-precision, non-destructive documentation tools, such as laser scanning, there is a significant degree of estimation that takes place in photogrammetry; a logic that suggests a special kind of 'interpretative' quality. This has to do, in the first place, with the relative inconsistency of the primary photographic material that is used in the digital reconstruction process. Photography is highly sensitive to the shifting environmental light conditions that are to be found in open-air and non-studio spaces, while it is also subject to a variety of photographic glitches that usually originate from reflective, transparent or complex surfaces. The algorithmic architecture of photogrammetric software, based on the fundamental rules of trigonometry and stereoscopy, tackles these inconsistencies with an estimative approach, attempting to reconstruct the original object by closing gaps, bridging inconsistencies and filling up whatever information has been accidentally left out during the photographic documentation. This has the result of creating a number of divergences from the actual three-dimensional topology of the original object. Often these divergences take the form of structural distortions, or even empty holes on the digital surfaces that make up the resulting $3 \mathrm{D}$ file. One could easily suggest that the reconstructed digital objects gain a 'ghostly' character from this algorithmic process, inherently imperfect, inevitably incomplete.

It is true that many of these faults can be prevented through the careful and experienced planning and execution of the documentative part of the process. This is often the case for scientific applications of the technique, although - as already explained - the limits of the photographic apparatus and the contingencies created by an uncontrolled environment will inevitably introduce inaccuracies.

The deficiencies of photogrammetry would not concern us further, were it to be solely dealt with within a professional scientific context. However, photogrammetry is a digital technique that has recently become increasingly popularized to the general public through a number of proprietary and open source software platforms and even more so through the launch of several mobile apps. In many cases, these apps or desktop software platforms simplify the overall process through accessible interfaces and playful instructions while, most importantly, outsourcing the demanding computational work that photogrammetric reconstruction requires to the digital Cloud. It is important to 
note here that this momentum of democratization in photogrammetry has been closely related to another field of the (post) digital market (Campbell et al. 2011). This is none other than the expanding industry of inexpensive desktop $3 \mathrm{D}$ printers and the variants of plastic-based filament materials that these machines use as consumables. Marketed to a broad public of non-professionals as a hobby aligned to the general character of the 'makers culture', cheap desktop computer-aided-manufacturing has been increasingly entering classrooms, workshops and households alike over the last decade. As this target group of customers is not necessarily familiar or experienced with 3D design, the printable content comes, more often than not, from online repositories that provide open source, free, or payable 3D models, or simple template-based apps that let their users customize already existing designs.

Given these limited options, it is obvious how simplified photogrammetry applications have strategically infiltrated this maker-culture industry as a tool that allows for a more intimate and interactive audience engagement with digital crafting and desktop manufacturing: the ease of reconstructing a familiar physical object in digital form through the common practice of taking photographs with a smartphone adds greatly to a personalised creative and productive experience, stirring the cultural fantasy of a 'cloning' type of mechanical reproduction. What is important to note here is that the popularisation of similar maker-culture tools and practices provides a novel context to think about the current state of technological appropriation and reproduction, which calls for an overall updating of our theoretical, cultural and legal understandings. Needless to say, it was also inevitable that such techniques would diffuse into contemporary art practices, not only because of their growing cultural relevance, but also as they are tools that make accessible and sustainable production techniques that were until recently only offered as expensive services by specialised rapid-prototyping studios or through acquiring unapproachable unattainable industry-grade equipment.

However, one must ask what is the nature of usage of such digital production techniques by the general public? Experienced users and amateurs of digital technological trends have been using such accessible 3D reconstruction applications for various purposes and for a plethora of subjects. A typical search on 3D model online-sharing repositories (e.g. Scan The World, Sketchfab and many more) reveals a focus on themes such as small-scale design objects or knickknacks, human portraits, sleeping pets, as well as museum exhibits or public sculptures. It is true that, apart from the engaging process of constructing such digital objects, the actual cases of (re)usage of such 3D files in further creative projects remains a study that has still to be pursued in a critical way. However, in most cases it is easy to obtain some information on the files' popularity by, for example, consulting download-count statistics or commentposts showcasing derivative projects. As mentioned above, downloading such files for $3 \mathrm{D}$-printing is a possible type of usage, speculating that mixing these $3 \mathrm{D}$ 
files in visual compositions, gifs or other type of digital animations is another possible application. However, the technical expertise needed to manipulate such formats, especially in contrast to common bitmap images or sound files, renders debatable the degree of utility or relevance of such digital 'offerings'. It would appear that, for the moment, the capturing, sharing and collection processes of casual photogrammetric files remains an end in itself for the casual user, driven by the enthusiasm and relative ease, attributed to the non-professional usage of the technique. When it comes to artistic production, on the other hand, the subjects and applications of the photogrammetric technique can be considered more broad and ambitious in relation to their subjects and scope. In recent years, artists have been increasingly using photogrammetry as an alternative to custom-made or ready-made 3D models, usually welcoming the technological limitations (or working around them) in order to either use its products as intermediate stages for analogue sculptural production (as mere references or 3D-printed artefacts), or as elements for the composition of digital videos and interactive or virtual narratives. ${ }^{49}$

If the general or specialized audiences consider the current (or better inherent) technical limitations of photogrammetry as a small price to pay for getting one step closer to the productive emancipation promised by the imaginary of the desktop-industrial-revolution, 'Future Bestiary' embraces them wholeheartedly. For the work, the three-dimensional faults and structural distortions are employed in a contemplative and critical manner, towards the introduction of a fluid, impartial and transformative aesthetic that rewires movements and hierarchies between the material and the digital. In other words, the technical slippage becomes part of an aesthetic style and a conceptual inquiry into the relationships between original and copy. And it does so by embracing the composite nature of this interpretative technical process: a synergy between bodily subjectivity, material complexity, technological limitations and algorithmic automation.

\section{Heritage implications}

This interpretative logic, inherent in the way we have been producing and experiencing representational images and reproductions since early modernity, is not novel or without historical precedent. However, its technical specificities call for a more multifaceted and entangled inquiry into the scientific, academic and legal discourse concerning the documentation and use of cultural heritage. For once we have to consider the implications of a general understanding of

${ }^{49}$ Examples of artists and filmographers utilizing photogrammetry include Morehshin Allahyari, Timur Si-Qin, Hito Steyerl, Clement Valla and Liam Young among many others. 
machine vision; the approach of algorithmic automation towards a novel kind of gaze (Steyerl 2016). This debate goes beyond any technical or physical limitations of our devices, since it is in fact a domain of computer engineering that implies design decisions of practical, but also ideological nature.

The special question that arises in the context of this article's premise, is what is the nature, status and implications of the production and distribution of these digital 3D objects created by photogrammetry (and by extension, through other 3D scanning techniques), when their subject is an object of cultural heritage? If it were possible to somehow filter the total amount of existent digital content that derives from three-dimensional documentation of cultural heritage, it would be possible to observe three basic categories: a) 3D models that are produced by institutional and scientific initiatives related to archaeology, preservation or museology, b) 3D models that were created by ordinary users (cultural heritage enthusiasts, museum-goers, tech enthusiasts and anyone that might experiment with such technologies as an alternative to taking normal photos or videos of historic artifacts) and c) artists and other cultural producers working within the creative industries (from 3D animators to cinematographers, graphic designers and so on).

To some degree, it is easy to distinguish the intentions behind each category, as well as to imagine the respective applications and even assume the level of quality and precision attributed to the outcomes produced in the different cases of researchers, the general public and art professionals. However, quality and precision aside, all of this digital material comprises an overall ecology of documentative representations that, as we suggest, maintains an unprecedented potentiality of current and future applications that can generate further reproductions not limited in the digital realm. Even if we keep the conversation about the implications of digital documentation, editing and distribution of cultural heritage within the limited and privileged discourse of artistic production, the cartography of such an ecology is still important, since it reveals an overall techno-cultural tendency of an ongoing, massive and complex project of digitisation of cultural heritage. This process is at the same time both 'accidental' and systematic, bringing together the activity of the general public, of institutional endeavours and corporate forces. Some illustrative projects within this trend, showcasing theoretically contrasting ideologies and attitudes towards their subject, are the totalitarian efforts of the Google Arts \& Culture project (spanning from digitisation to $3 \mathrm{D}$ reconstruction of cultural artefacts, the production of virtual versions museum spaces, mass archiving and online content curation) and the Perpetuity | Palmyra project, which attempted to reconstruct a 3D model of the Arch of Triumph of Palmyra destroyed by ISIS in 2015 , by using a photogrammetry field with photographs taken by tourists before the event. ${ }^{50}$

${ }^{50}$ See https://the-arckives.org. Last accessed July 2019. 
How can one evaluate such a technical process in relation to what we already know about media distribution and reproduction? It would seem that it becomes even more complex on a technical level. It is revealing to just take note of one practical aspect of the photogrammetric technique: once a digital photogrammetric reconstruction is obtained in the form of a 3D file, the collection of digital photographs that were used as primary material can be (and usually are) discarded. This leaves us with a three-dimensional reproduction with no familiar documentation source, as it would be the case with photography for example, which has already been debated broadly in relation to the question of its potential to mechanically reproduce cultural material. ${ }^{51}$ What we obtain here is a 'unique object', with no familiar traceable past, a generated mathematical abstraction brought into existence only by a visual reference to an original counterpart, a reference that does not exist anymore. In contrast, this dynamic format of the $3 \mathrm{D}$ file provides us the possibility for a new series of reproductive activities, such as the translation to new material objects through computer-aided-manufacturing discussed above.

Perhaps, this idiosyncratic character of the photogrammetric technique, its inability to provide us with a) exact technical representations b) familiar media formats that have already been debated in relation to their reproductive status and c) its fluent ability to generate new reproductive potentials, could be a crucial opportunity for a discussion beyond the technical and institutional characteristics that the issue of usage of cultural heritage has been officially built upon. If our technical apparatuses can bring us with such ease in front of extremely hybrid and transformative examples of the original-copy scheme, then questions of technical resolution and quality, of professional and profitoriented usage and of cultural heritage could start being replaced with more broad and fundamental ones. What are the novel cultural potentials of our digital reproductions? To whom do they belong intellectually and legally as cultural material? What does the possibility for their further accessibility and dissemination mean in a practical sense?

\section{Legal implications}

Petros Moris' artwork 'Future Bestiary' perfectly reflects this artistic practice of using the image of pre-existing culturally significant works of art and re-contextualizing them. For Petros, the exploration of the Structure-fromMotion photogrammetric technique and its incorporation into his creative process transgresses the boundaries between materiality and immateriality,

51 See for example the seminal essay by Walter Benjamin, 'The Work of Art in the Age of Mechanical Reproduction'. 
challenging the notions of reproduction and authenticity as traditionally perceived by legal scholars.

Within this framework, some interesting questions regarding the interpretation of heritage accessibility from a legal standpoint may arise. What are the implications of the emergence of these contemporary artistic practices for cultural heritage appropriation? How does the Greek legislation on antiquities and cultural heritage deal with this issue in general $?^{52}$ In other words, could a hypothesis for increased cultural heritage preservation along with that of cultural heritage accessibility for artistic purposes be envisaged?

Greek Archaeological Law 3028/2002 "on the protection of antiquities and cultural heritage in general" broadens the scope of cultural heritage protection as it provides an extensive definition of cultural objects as "testimonies of the existence and the individual and collective creativity of human kind" (A.2) and it covers manifestations of both tangible and intangible cultural heritage.

Article 46 of the Greek legislation regulates the accessibility and use of "monuments" 53 and sites. According to the paragraphs 4 and 5 of article 46 of the Archaeological Law 3028/2002, a previous permission granted by the Ministry of Culture is required for the production, reproduction and dissemination to the public of impressions, copies or depictions of monuments belonging to the Public Sector, or immovable monuments that are located within archaeological sites and historical places or are isolated, or movable monuments that are kept in museums or public collections, in any way and by any means whatsoever, including ICT. Such permission is granted to natural or legal persons for a fee paid to the Fund of Archaeological Proceeds (TAP) upon decision of the Minister of Culture, while the decision also specifies the temporal validity of the permission, the terms on which the permission is granted and the fee that must be paid. The production, reproduction and use of the aforementioned goods for other purposes, such as artistic, educational or scientific

52 Archaeological Law 3028/2002 on the protection of antiquities and cultural heritage in general, Government Gazette, (hereinafter FEK) A' 153. For an official translation of this law into English see: http://www.unesco .org/ulture/natlaws/media/pdf/greece/grelaw_3028_engtof.pdf. Last access April 2019.

53 The Greek legislator used the term "monuments", a term referring to memory, to describe ancient and other protected tangible cultural objects. According to Article 2, sub para. (b), by "monuments" are meant cultural objects which constitute material evidence and belong to the country's cultural heritage, whose special protection is called for. Monuments are divided into ancient and modern (or "recent" in the official translation) (i.e., those later than 1830), and also divided into "immovable" and "movable". 
purposes, is again allowed for a fee paid to TAP, however, the fee can be waived upon decision of the Minister of Culture ${ }^{54}$ (See also Tsiavos this volume).

When reading the relevant regulatory framework, two main considerations arise with respect to the creative use and reuse of cultural heritage content for Greek monuments and other cultural goods. Firstly, it is obvious that there is a state-centric character for heritage protection resulting from historical reasons related to the centralized cultural policy tradition of the country. This conservative approach exclusively establishes antiquities and other protected cultural goods as a privileged symbolic foundation for national identity (Voudouri 2010). Under this framework, the mediation of ICT tools and methods can be interpreted as being in line with this state-centric vision for cultural heritage only if it is used for preservation, protection, educational and research purposes. The creative and artistic aspect of re-purposing and re-using digital cultural heritage content is clearly underestimated - if not excluded - from the scope of the national legislator. Even where the legislator acknowledged the primacy of heritage's social function, ${ }^{55}$ the limitations of experiencing heritage by individuals and communities is still a challenging issue, affecting the essence of heritage as a public good to be fully accessed and enjoyed by everyone. ${ }^{56}$

Secondly, the existing rules on the use of digital technologies for the reproduction, use and preservation of cultural heritage content is obviously out-

${ }^{54}$ Common Ministerial Decree no 81397/2199/21-09-2005 provides a number of dispositions regulating the permission procedure for using cultural heritage content digitally. The Public Sector and the TAP are excepted from paying fees for any kind of use, however the relevant permission by the Ministry of Culture should be granted in any case.

${ }^{55}$ Within the Archaeological Law 3028/2002, it is evident that the preservation is not understood as an end in itself. See mainly A.3 on the content of the protection, A.45 on museums and A.46 on access to end use of monuments and sites.

56 With regards to the re-use of Public Sector Information (PSI), despite the fact that Directive 2013/37/EU, amending PSI Directive 2003/98/EC, is or at least was meant to be a determinant pillar of the European Union's open data strategy, the amended PSI Directive permitted the contractual restriction on the commercial reuse of public domain works which have been digitised under a Public Private Partnership (PPP). The contractual restrictions are in principle restricted to ten years but may run longer as long as they are subject to review. See Pekel, Fallon \& Kamenov, Public Sector Information in Cultural Heritage Institutions, June 2014, https://www .europeandataportal.eu/sites/default/files/library/201406_public_sector _information_in_cultural_heritage_institutions.pdf. Last access July 2019. 
dated. Since the beginning of the $20^{\text {th }}$ century, the development of services supporting the implementation of digital technology in the cultural heritage sector has significantly contributed to bridge the gap between ICT research and cultural heritage. Beginning with the introduction of digital technology into the infrastructure of cultural institutions and the digitisation of cultural heritage content, this process eventually led to considerations for updating the regulatory framework regarding the digital content use. These considerations should not only be limited to educational or research purposes; the artistic factor should also be taken into account. A most interesting possibility raised by the digitisation of cultural heritage content is transmitting the value of original sources and finding sophisticated ways to reintroduce the past into everyday life. Not only serving students, scholars, and educators, but also inspiring new artists and fostering future interest in cultural heritage collections is critical to the longevity and relevance of cultural heritage itself.

\section{Moving forward}

It is necessary to move away from the official approaches to heritage that exclusively view monuments as a privileged symbolic foundation for national identity and it is essential to strongly support an open, accessible cultural heritage that will serve as an inspirational pole for contemporary artistic practices. It is urgent that we explore new ways of collectively rethinking our approach to reproduction, storage and sharing of artworks and cultural heritage in the $21^{\text {st }}$ century. ${ }^{57}$ New technologies provide great opportunities so that cultural heritage be more accessible, and cultural experience be more meaningful. It is urgent that the dialogue opens up globally by offering opportunities for creative collaboration and coexistence between the ancient and the contemporary, between the past and the future, the original and the copy, by constructing realistic legal licencing systems that promote accessibility, reuse, and thus creativity.

57 The ReACH project (Reproduction of Art and Cultural Heritage) was a valuable initiative coordinated by the Victoria and Albert Museum in partnership with the Peri Foundation, the Louvre Museum, the Smithsonian Institute and other key research partners, which resulted in the production of a Declaration embracing digital technologies and offering new ways to produce, store and share museum and heritage assets, see https://www.vam .ac.uk/research/projects/reach-reproduction-of-art-and-cultural-heritage. Last access June 2019. 

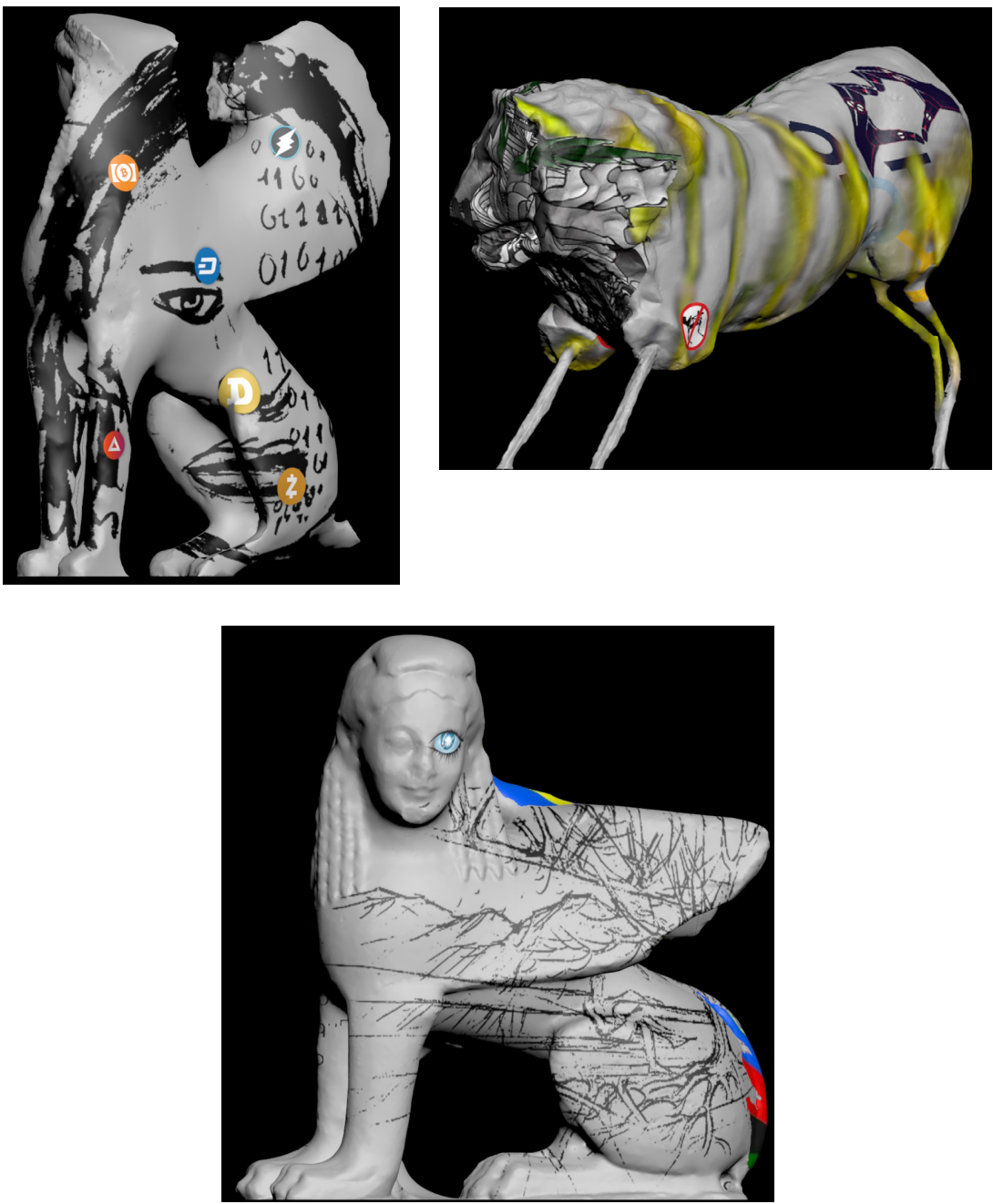

Figures 4, 5, 6: Petros Moris, Future Bestiary, 2019, HD video projection (5:00) (Source: PM). 


\section{Bibliography}

Al-Ruzouq, R. (2012). Photogrammetry for archaeological documentation and cultural heritage conservation. In Da Silva, D. C. (Ed.), Special applications of photogrammetry (pp. 97-110). Retrieved April 7, 2019 from www intechopen.com.

Campbell, T., Williams, C., Ivanova, O. \& Garrett, B. (2011). Could 3D printing change the world?: Technologies, potential and implications of additive manufacturing. Strategic Foresight Report, October. Retrieved April 7, 2019, from https://www.atlanticcouncil.org/wp-content/uploads /2011/10/101711_ACUS_3DPrinting.PDF.

De Clippele, M.-S. \& Lambrecht, L. (2015). Art law and balances: Increased protection of cultural heritage law vs. private ownership: Towards clash or balance? International Journal of Cultural Property, 22, 259-278. DOI: https://doi.org/10.1017/S0940739115000119.

Eschenfelder, K. R. \& Caswell, M. (2010). Digital cultural collections in an age of reuse and remixes, First Monday, 15(11). Retrieved April 7, 2019, from https://firstmonday.org/article/view/3060/2640.

Hamilton, G. \& Saunderson, F. (2017). Open licensing for cultural heritage. London, United Kingdom: Facet Publishing.

Ioannides, M., Chatzigrigoriou, P., Bokolas, V., Nikolakopoulou, V., Athanasiou, V., Papageorgiou, E., ... \& Sovis, C. (2017). Educational creative use and reuse of digital cultural heritage data for Cypriot UNESCO monuments. In: M. Ioannides M., E. Fink, A. Moropoulou, M. Hagedorn-Saupe, A. Fresa, G. Liestøl ... \& P. Grussenmeyer (Eds.), Digital heritage: Progress in cultural heritage: Documentation, preservation, and protection. 6th International Conference, EuroMed 2016, Nicosia, Cyprus, October 31 - November 5, 2016, Proceedings, Part I. Lecture Notes in Computer Science, vol. 10058 (pp. 891-901). Cham, Switzerland: Springer.

Kuan, C. (2014). 7 Lessons learned for digital culture. In H. Din \& S. Wu (Eds.), Digital heritage and culture: Strategy and implementation (pp. 41-52). Singapore, Singapore: World Scientific. DOI: https://doi .org/10.1142/9789814522984_0004.

Law 3028/2002. On the protection of antiquities and cultural heritage in general. Retrieved November 20, 2019, from https://www.bsa.ac.uk/wp-content /uploads/2018/11/Archaeological-Law-3028-2002.pdf.

Newell, J., Lythberg, B. \& Salmond, A. (2012). Old objects, new media: Historical collections, digitization and affect. Journal of Material Culture, 17(3), 287-306.

Panezi, A. (2018). Europe's new renaissance: New policies and rules for digital preservation and access to European cultural heritage. Columbia Journal of European Law, 24(3), 596-611. 
Pary, R. (2007). Recoding the museum: digital heritage and the technologies of change. London, United Kingdom: Routledge.

Ruthven, I. \& Chowdhury, G. G. (Eds.). (2015). Cultural heritage information: Access and management. London, United Kingdom: Facet Publishing.

Steyerl, H. (2016). A sea of data: Apophenia and pattern (mis-)recognition. E-Flux Journal, 72. Retrieved April 7, 2019 from https://www.e-flux.com /journal/72/60480/a-sea-of-data-apophenia-and-pattern-mis-recognition/.

Voudouri, D. (2010). Law and the politics of the past: Legal protection of cultural heritage in Greece. International Journal of Cultural Property, 17, 547-568.

Wachowiak, M. J. \& Karas, B. V. (2009). 3D scanning and replication for museum and cultural heritage applications. Journal of the American Institute for Conservation, 48(2), 141-158.

Younan, S. (2015). Poaching museum collections using digital 3D technologies. Journal of Science and Technology of the Arts, 7(2), 25-32. 